\title{
FRACTURE-DISLOCATION OF THE LUMBO-SACRAL SPINE WITH CAUDA EQUINA LESION
}

\author{
Report of Two Cases
}

\author{
P. Dewey, Wagga Wagga, Australia, and P. S. H. Browne, Oswestry, England \\ From the North Staffordshire Hospital Centre, Stoke-on-Trent, England
}

Traumatic dislocation of the fifth lumbar vertebra on the sacrum is rare. Watson-Jones (1940) described " an unusual and instructive case" of lumbo-sacral dislocation with a cauda equina lesion, in which the patient eventually died from ascending urinary tract infection. Evans (1959) described another case associated with a fractured femur. The dislocation was missed at first and intractable bed-sores drew attention to the cauda equina lesion.

\section{CASE REPORTS}

Case 1-A woman aged thirty-one was involved in a road accident while a back seat passenger in a car. She was shocked and complained of pain in the back and of "pins and needles" down both legs. Examination revealed a boggy haematoma in the lumbo-sacral region with some overlying skin abrasions. There was a palpable "step" from the spine of the fifth
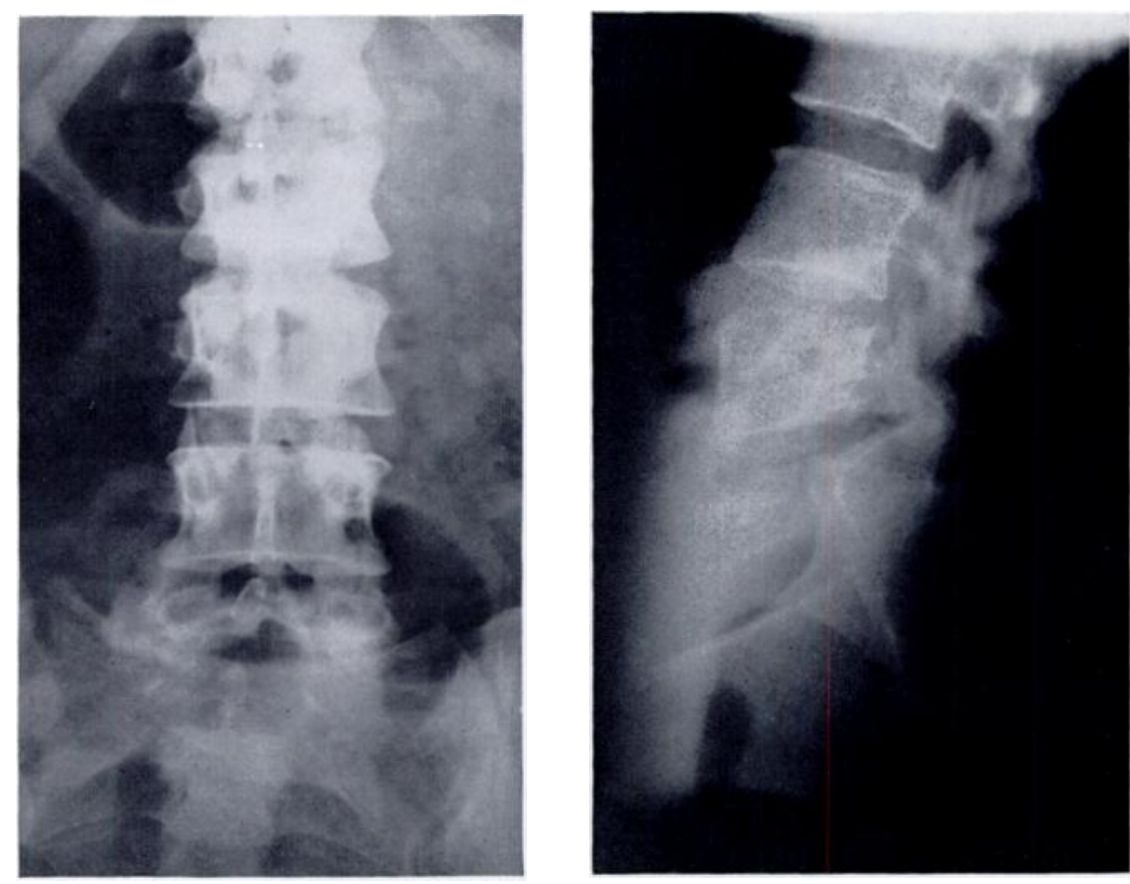

Fig. 1

Case 1-Antero-posterior and lateral radiographs of the lumbar spine showing forward dislocation of the fifth lumbar vertebra on the sacrum, and posterior wedging of the body of the fifth lumbar vertebra. Fractures of the transverse processes of the vertebrae above the lesion are seen on the left side.

lumbar vertebra to the sacrum. Both knee jerks were brisk, both ankle jerks absent, and both plantar responses unobtainable. There was complete anaesthesia in the distribution of the first sacral nerve and of the nerves distal to it. Motor power was difficult to estimate initially, but weakness of the right foot and ankle was demonstrated. The power in the left foot and 
ankle was considered to be normal. The patient developed retention of urine, necessitating an indwelling catheter.

Radiographs showed forward dislocation of the fifth lumbar vertebra on the sacrum (Fig. 1). There was disruption of the spine and pedicles and posterior wedging of the body of the fifth lumbar vertebra. Fractures of the transverse processes of the other lumbar vertebrae were also present.

Reduction was attempted by traction (Watson-Jones 1940) with the hips flexed to 100 degrees and the buttocks just off the bed, but without success. Two days later an attempt was made to reduce the dislocation by manipulation under anaesthesia, but this also failed. Open reduction was considered inadvisable on account of the poor condition of the overlying skin. Traction was maintained for two weeks.

Progress-During the next two months the patient's general condition improved. Sensibility returned to the areas supplied by the first sacral nerve on both sides, but she remained anaesthetic in the areas supplied by the second sacral and more distal nerves. Muscle power improved in the legs but she required double irons and outside $\mathrm{T}$-straps to compensate for residual paralysis of the peroneal muscles. There had been no return of bladder or bowel function.

Ten weeks after the injury she was transferred to Manchester Royal Infirmary under the care of Mr D. Lloyd Griffiths, and we are grateful for a report on her six months after the initial injury. Urinary and bowel function were then normal. She walked with a waddling gait but managed her own shopping and housework. There was considerable restriction of movement of the lumbar spine. Motor power in the lower limbs was normal except for the peroneal muscles, extensor hallucis longus and extensor digitorum longus. These muscles were of power 3 in the left leg and power 1 in the right leg (Medical Research Council grading). There was hypoaesthesia over the outer side of the left foot and ankle and almost complete anaesthesia over the outer side of the right foot and ankle. Both ankle jerks were absent.

Case 2-A man aged thirty-three was struck by a fall of rock while kneeling at work in a mine.

He was shocked and complained of pain in the back and left leg. In addition to his back injury there was a comminuted fracture of the shaft of the left femur and fractures of the right ankle and right hand. Examination revealed a haematoma in the lumbo-sacral region with a definite step from the fifth lumbar vertebra to the sacrum. There was impairment of sensibility in the areas of skin supplied by the first sacral nerve in both legs and saddle anaesthesia below this level. The muscle power and reflexes were difficult to assess because of the patient's other injuries, but an impression of weakness in both ankles and feet was gained.

Radiographs (Fig. 2) showed forward dislocation of the fifth lumbar vertebra on the sacrum, with a fragment displaced forwards from the upper surface of the body of the sacrum. There were also fractures of the transverse processes of the first. second and third lumbar vertebrae on the right side.

The patient was resuscitated, and the lumbo-sacral region of the spine was explored nine hours after the injury. The dislocation was seen and it was found to be irreducible without complete facetectomy. This would have made the spine very unstable. Decompression was therefore carried out by removal of the spinous processes of the fourth and fifth lumbar vertebrae, followed by excision of the lamina of the fifth lumbar vertebra and part of the fourth. The cauda equina was exposed and found to be kinked sharply backwards by the lower border of the lamina of the fifth lumbar vertebra. Remnants of the lumbo-sacral disc were lying sequestrated in the spinal canal. These were removed and the disc space explored.

The patient's condition deteriorated after operation and he died next morning. Necropsy showed the cause of death to have been extensive fat embolism.

Post-mortem dissection of the lumbo-sacral spine showed that the articular facets on both sides were locked and that even when all soft tissue had been removed manual reduction 
was impossible. The intervertebral disc was grossly disorganised and the annulus fibrosus torn cleanly from the vertebrae in several places. The anterior longitudinal ligament was intact and there was only a little haemorrhage anteriorly. The pathologist commented that this dislocation could easily have been missed during routine necropsy, particularly with the other gross injuries which could have caused the patient's death.
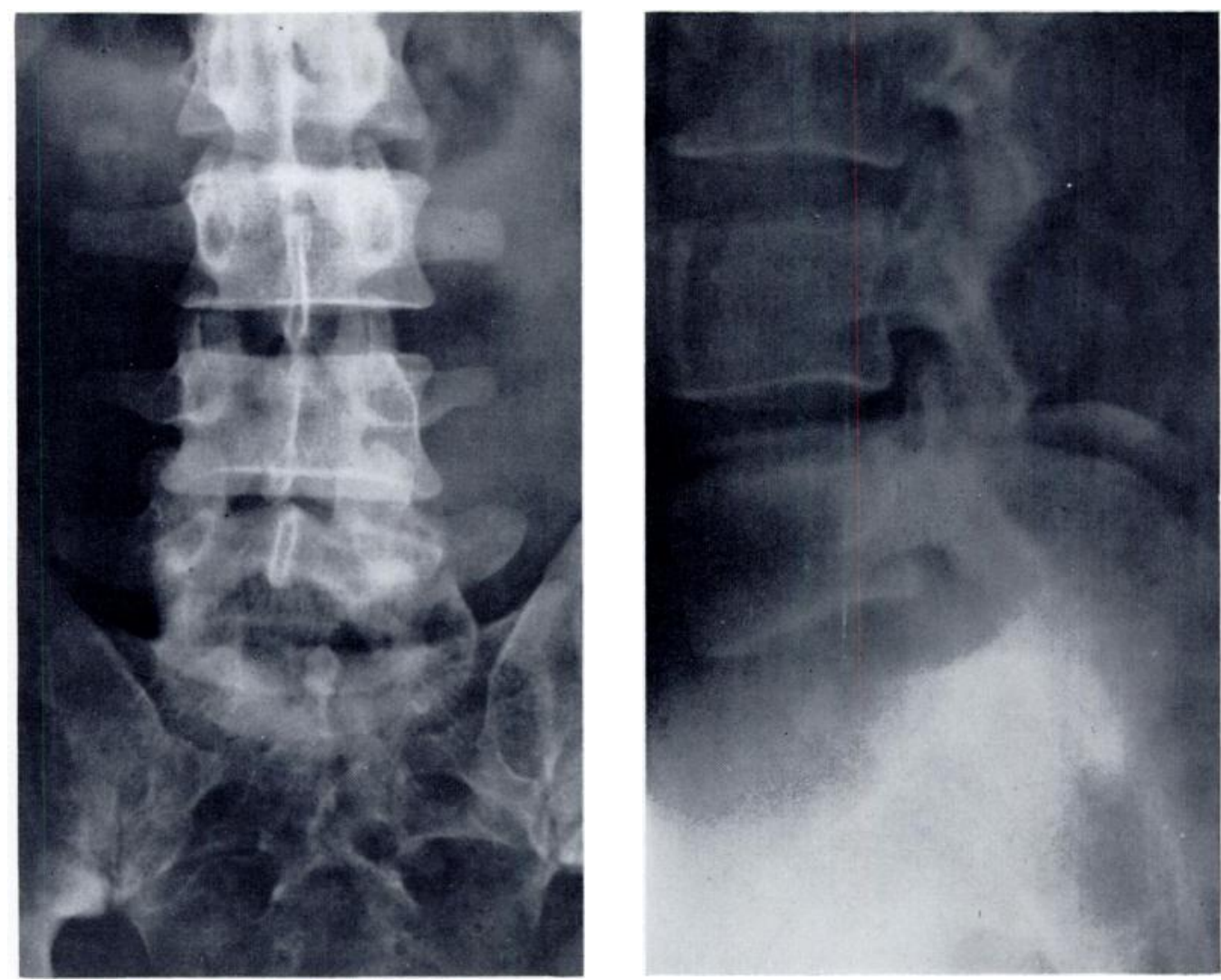

Fig. 2

Case 2-Antero-posterior and lateral radiographs showing forward dislocation of the fifth lumbar vertebra on the sacrum, with a displaced fragment from the upper surface of the body of the sacrum. Fractures of the transverse processes of the first, second and third vertebrae are seen on the right side.

\section{DISCUSSION}

Both these patients had received severe injuries, one from a motorway accident and the other from a mining accident, and both were very ill on admission to hospital. The first patient was treated by traction and manipulation. It is our impression that the patient's neurological signs were exacerbated by this treatment. Newman (1965) reported similar results after treating chronic lumbo-sacral subluxation by traction.

The second patient was treated by operation. The cauda equina was found to be compressed by disrupted lumbo-sacral disc material and by the lamina of the fifth lumbar vertebra. Laminectomy was necessary to remove both compressing agents. The lumbo-sacral joint was found to be locked in the dislocated position, both at operation and at necropsy, when it was necessary to do complete facetectomy to reduce the dislocation.

Watson-Jones (1940) considered the mechanism of this injury to be forced hyperextension. We find it to be difficult to be as precise as this. Both our patients had fractures of the transverse processes of the lumbar vertebrae above. Both were in a confined space with flexed knees 
and hips fixing the pelvis when they were injured. We suggest that the lesion resulted from a severe force applied to the lumbar spine, shifting it forward on the immovable sacrum, and so producing the dislocation.

In view of the difficulty in reducing this dislocation, even when operation was performed, and the fact that neurological improvement occurred after incomplete reduction with conservative treatment, we suggest that patients with this lesion should be treated initially by simple pelvic traction.

\section{SUMMARY}

1. Two cases of fracture-dislocation of the spine at the lumbo-sacral level are reported.

2. One patient was treated conservatively and survived, with a cauda equina lesion which is now recovering. One patient was treated by operative decompression and died of fat embolism. 3. The lumbo-sacral joint is locked in the dislocated position.

We are grateful to Mr Denys Wainwright and to Mr A. K. Mitting for permission to report their cases. We should like to thank Mr D. Lloyd Griffiths and Mr R. S. Phillips for a follow-up report on the first patient.

\section{REFERENCES}

Evans, E. M. (1959): Some Recent Studies in Spondylolisthesis. Journal of Bone and Joint Surgery, 41-B, 430. Newman, P. H. (1960): A Clinical Syndrome Associated with Severe Lumbo-sacral Subluxation. Journal of Bone and Joint Surgery, 47-B, 472.

Watson-Jones, R. (1940): Fractures and Other Bone and Joint Injuries. First edition, p. 641. Edinburgh: E. \& S. Livingstone. 